\title{
Una aproximación a las emociones positivas generadas en las relaciones intergeneracionales: principales determinantes que inciden en la felicidad y bienestar de los mayores
}

\author{
Antonio Luis Martínez-Martínez ${ }^{1}$ \\ Marcos Bote Díaz \\ Juan Antonio Clemente Soler ${ }^{3}$
}

1 Profesor doctor. Departamento de Sociología Universidad de Murcia. Campus de Espinardo. 30100. Correo electrónico: amm19000@um.es. https://orcid.org/0000-0001-8031-9158

2 Profesor contratado Doctor. Departamento de Sociología. Universidad de Murcia. Campus de Espinardo 30100. Correo electrónico: mbote@um.es. https://orcid.org/0000-0002-9178-3105

3 Profesor doctor. Departamento de Sociología. Universidad de Murcia Campus de Espinardo 30100. Correo electrónico: juanantonio.clemente@um.es. https://orcid.org/0000-0003-2272-6219

Fecha de recepción: 22/02/2020. Fecha de aceptación: 20/08/2020. 
Una aproximación a las emociones positivas generadas en las relaciones intergeneracionales: principales determinantes que inciden en la felicidad y bienestar de los mayores

\title{
RESUMEN
}

La literatura sobre los efectos positivos de las relaciones intergeneracionales, tanto para personas mayores como para jóvenes y adultos, es extensa. Sin embargo, en pocas ocasiones se han presentado evidencias sobre los beneficios de este tipo de relaciones más allá del efecto integrado de las redes de apoyo social. El presente estudio tiene por objetivo dilucidar el efecto de la existencia de relaciones intergeneracionales familiares en el estado de felicidad de las personas mayores de 65 años en España. Para ello se ha usado el barómetro del CIS 3109 de septiembre de 2015, que analiza el efecto de las relaciones y convivencia con familiares menores de 35 ańos en la felicidad de las personas mayores, tanto de forma aislada, como en combinación con otras variables. Los resultados muestran cómo convivir o tener relaciones con personas menores de 35 años no tiene un efecto significativo sobre la felicidad de las personas mayores. Factores como el género, el estado civil, el estatus socioeconómico y, sobre todo, la salud, tienen un mayor impacto en la escala de felicidad percibida.

Palabras clave: felicidad, relaciones intergeneracionales, estado de salud.

\section{An Approach to the Positive Emotions Generated in Intergenerational Relation- ships: Main determinants that affect the happiness and well-being of the elderly}

\begin{abstract}
There is extensive literature on the positive effects of intergenerational relationships for both older people and young people. However, in very few cases evidence of the benefits of this type of relationships beyond the integrated effect of support social networks has been presented. This study aims to elucidate the effect of the existence of family intergenerational relationships in the state of happiness of people over 65 in Spain. To do this, we used the CIS 3109 Barometer of September 2015, in order to analyze the effect of relationships and coexistence with family members under 35 years in the happiness of the elderly, both in isolation, and in combination with other variables. The results show that cohabiting or having relationships with people under 35 does not have a significant effect on the happiness of the elderly. Factors such as gender, marital status, socioeconomic status and, above all, health, have a greater impact on the scale of perceived happiness.
\end{abstract}

Keywords: happiness, intergenerational relations, state of health. 


\section{INTRODUCCIÓN}

Ejercer de abuelo es gratificante y placentero, ya que experimentan elevados niveles de satisfacción, alegría, felicidad, sentimientos de juventud y utilidad (Osuna, 2006). En este sentido Marín y Palacio (2015) manifiestan, que la abuelidad supone una nueva oportunidad para revivir únicamente las experiencias positivas de la crianza, liberándoles esta vez de las obligaciones y responsabilidades parentales. De otra parte, debemos puntualizar que también se han analizado en profundidad los beneficios de las relaciones intergeneracionales, y estimamos conveniente focalizar nuestra atención, aunque sea someramente, en las emociones positivas que conllevan las citadas relaciones, estrechamente vinculadas a los efectos positivos experimentados por abuelos y nietos. En este sentido, Sanz, Mula y Moril (2011) manifestaban que la transmisión de valores adquiere una gran notoriedad, permitiendo consolidar las relaciones basadas en la cercanía, afecto y carińo, y resultan esenciales para lograr el correcto desarrollo psicológico y social de los menores. También Pinazo, Bendicho y Company (2008) estiman que el contacto asiduo entre abuelos y nietos fomenta la cooperación, proporciona una comunicación más cercana y permite compartir sentimientos.

\section{Aproximación conceptual a las emociones humanas}

Ingentes investigaciones han estudiado el ámbito de las emociones humanas y la importancia que adquieren en nuestra vida, aportando múltiples teorías desde todas las disciplinas sociales (antropología, sociología, psicología, lingüística etcétera). Por ende, estimamos idóneo, en primer término, ofrecer algunas de las definiciones más notorias del concepto de emoción acuñado por algunos autores. Fernández (2011) define las emociones como procesos mentales básicos pero al mismo tiempo complejos, pues engloban aspectos neurofisiológicos, bioquímicos y psicológicos. Esta autora destaca que los sentimientos equivalen a la parte cultural de las emociones, una vez están codificadas y personalmente definidas. Precisamente Marina (2006) las describe como sentimientos breves, pero que aparecen de forma abrupta y se manifiestan a través de respuestas fisiológicas tales como palpitaciones, temblor, palidez o rubor. En referencia al intervalo temporal de las emociones, Filliozat (2007) también coincide con el anterior autor.

Sin embargo, Denzin (2009) nos ofrece una definición más filosófica: «Una experiencia corporal viva, veraz, situada y transitoria que impregna el flujo de conciencia de una persona, que es percibida en el interior de y recorriendo el cuerpo, y que, durante el trascurso de su vivencia, sume a la persona y a sus acompańantes en una realidad nueva y transformada, la realidad de un mundo constituido por la experiencia emocional» (p. 66). Lawler (2001) además establece una distinción 
entre emociones globales y específicas. Las primeras son consideradas respuestas involuntarias y no condicionadas, y resultan del acaecimiento de situaciones inesperadas; por el contrario, en las segundas, las personas a priori ya han asociado una emoción a un objeto, sujeto o circunstancia determinada.

A partir de las definiciones descritas, podemos establecer que las emociones conllevan, de una parte, una dimensión personal que representa una valoración o reacción positiva o negativa hacia algo o alguien y, de otra, una elevada dimensión social. En este sentido Stryker (2004) manifiesta que la expresión de una emoción concreta no solamente dependerá de la conducta que esa persona adopta, sino también del grado en que dicha actitud repercute en su índice de bienestar, necesidades, metas, objetivos etcétera. También las emociones dependerán de las expectativas puestas en las interacciones sociales y, en función de su índice de cumplimiento, serán positivas o negativas (Burke y Stets, 2009).

Antes de proceder a desarrollar este acápite, consideramos oportuno llevar a cabo una distinción suscinta entre emociones y sentimientos, pues se emplean asiduamente de manera indistinta. Cierto es que son dos elementos interconectados y dependientes, pero los sentimientos corresponden a la parte nombrable de las emociones, cuando estas están delimitadas. Al respecto, Damasio (2006) estima que las respuestas fisiológicas de las emociones obviamente se vinculan al cuerpo, mientras que los sentimientos a la mente. También Scribano (2012) manifiesta que las emociones son estados corporales.

A continuación desarrollaremos las diferentes categorías en las cuales pueden subdividirse las emociones. Por una parte podemos encontrar las emociones primarias, que corresponden a la cólera, la alegría, el miedo y la tristeza; y las secundarias, que se refieren al amor, la sorpresa, la vergüenza y la aversión, aunque autores como Turner (1999) añaden a esta última división otras como la culpa, el resentimiento, la decepción y la nostalgia. Bericat (2012) considera a las emociones primarias como respuestas universales, fisiológicas, y biológica y neurológicamente innatas, y a las secundarias con un elevado grado de condicionamiento social y cultural.

Por su parte, Jasper (2011) establece una división diferente. En primer término, considera los denominados impulsos corporales, como el deseo sexual; en segundo término, las emociones reflejas, que corresponden a aquellas que se producen durante un breve lapso, como la ira, el miedo o la alegría. En tercer lugar, los humores o estados afectivos, que se producen con bajos niveles de intensidad pero pueden perdurar; $y$, finalmente, las emociones reflexivas, que aluden, de una parte, a los sentimientos positivos profesados hacia un sujeto, como el amor, el respeto y la confianza, y también a aquellos cargados de un fuerte componente moral y que implican un evidente grado de aprobación o rechazo. 
Debemos considerar que las emociones adquieren una notoria complejidad. Al respecto, Bericat (2012) estima que las emociones no deberían considerarse como meras respuestas mecánicas o fisiológicas producidas por las circunstancias del entorno, sino que se producen también debido a otros factores, como el objeto que las suscita, la valoración consciente o inconsciente de los elementos que nos rodean, el grado de expectativas ante determinadas situaciones, la identidad activa y, finalmente, la manera en que se identifica a otras personas, grupos o colectivos. De otro lado, es necesario establecer una distinción clara entre emoción y cognición, y entre emoción y otros estados afectivos como sentimientos, pasiones y afectos (Guedes y Álvaro, 2010). Destacamos que las terminologías de emoción y cognición están incardinadas, y la primera influye notablemente en el sistema cognitivo - especialmente en la memoria - y nos permite recordar determinados episodios de nuestra vida y obviar algunos (Damasio y otros, 2001).

Desde las ciencias sociales, como ya mencionamos, se han escrito muchas teorías referidas al ámbito de estudio de las emociones. Luna (2010), basándose en Hochschild (1990), las subdivide en tres grupos diferenciados: orgánico naturalista, construccionismo no radical y constructivismo radical, en función del grado atribuido de componentes biológicos o culturales que presenten las bases que las sustentan. En las investigaciones de Smith y Schneider (2009) se clasifican desde una perspectiva determinista, construccionista social, y finalmente desde una visión interaccionista social. Gross y Feldman (2011) evalúan los niveles de regulación de las emociones basándose en la naturaleza de las mismas y clasificándolas en emociones básicas, modelos evaluativos, modelos de construcción psicológica y finalmente modelos de construcción social. Desde la teoría de la atribución desarrollada por Lawler, Thye y Yoon (2008), las emociones no dependen en exclusividad de la conducta manifestada ni del hecho generador, sino de las representaciones o atribuciones causales llevadas a cabo por cada individuo.

De otra parte, para Belli e Íñiguez (2008), al considerar a las emociones como expresiones corporales que se manifiestan a través del lenguaje se evidencia una notoria carga discursiva, motivo por el cual se analizan ampliamente desde la psicología y específicamente desde la psicología social de la emoción y propiamente desde los discursos de la emotividad. Por ende, desde el ámbito de la psicología social contemporánea, focaliza su atención en cinco áreas principales:

- La primera estudia el binomio emoción-lenguaje, y la comunicación oral es el nexo a través del cual se expresan los sentimientos (Good, Good y Fischer, 1988).

- La segunda otorga especial relevancia a los aspectos históricos y antropológicos, que sustentan la manifestación de las emociones (Harré y Stearns, 1995).

- La tercera analiza propiamente el discurso de las emociones (Edwards, 1999). 
- Una cuarta corriente, de carácter posconstruccionista, sobre la base de los trabajos de Íñiguez (2005), quien acuña el término de perfomatividad, considera que las emociones, al igual que las relaciones humanas, están constantemente redefiniéndose y reestructurándose.

- Finalmente una vertiente tecnocientífica, enmarcada en el proyecto de máquina afectiva (Brown, 2005; Michael, 2006).

\section{La felicidad como máxima expresión de bienestar}

Sin duda, la felicidad ha sido un concepto ampliamente analizado en la historia del pensamiento humano, y se considera el máximo exponente de las emociones positivas, al englobar múltiples dimensiones relativas al bienestar (logros, metas, aspiraciones, índices de satisfacción, estabilidad etcétera). Al respecto, Diener (2000) la considera un concepto universal y dotado de gran trascendencia, y ha sido ampliamente tratada en la filosofía oriental y occidental (Haidt, 2007). Al mismo tiempo, sin embargo, se trata de un concepto ambiguo desde una perspectiva científica, y dada su difícil clasificación y delimitación, se estima el empleo de otras acepciones tales como bienestar, bienestar psicológico, satisfacción. Prueba de ello son algunos autores como McMahon (2006), que la considera un concepto polisémico, al estar su definición y condiciones sometidas a criterios diferentes. Otro ejemplo es el trabajo de Arraga y Sánchez (2010), los cuales consideran que su complejidad reside en que se relaciona directamente con el sentido positivo de la vida, sentimientos de alegría y complacencia, y no solamente se define por los éxitos ya obtenidos, sino por los que se espera conseguir en breve.

A continuación procedemos a establecer una aproximación conceptual al término "felicidad», exponiendo de manera suscinta sus principales características y tomando en referencia a varios autores.

Alarcón (2006) la define con las siguientes palabras: «Es un estado de satisfacción, más o menos duradero, que experimenta subjetivamente un individuo en posesión de un bien deseado» (p. 8). El mismo autor, en una investigación posterior (2015), manifiesta que la felicidad está conformada por los sentimientos vivenciados que proporcionan satisfacción, y dota de suma relevancia a su dimensión subjetiva, lo que le permite establecer una medición de la felicidad en grados o niveles.

Otra acepción es la aportada por Beytía y Calvo (2011), quienes señalan que la felicidad es el grado mediante el cual cada persona aprecia su vida de manera positiva en la totalidad de dimensiones que la conforman. Esta definición considera que la felicidad constituye un concepto subjetivo.

Por otra parte, Russell (2004) manifiesta que los principales factores vinculados a la felicidad son la satisfacción con la vida, la alegría por vivir, la realización 
personal (autorrealización) y, finalmente, la ausencia de sufrimientos profundos. Basándonos en este autor podemos establecer cuáles son las dimensiones principales que propician los sentimientos positivos:

- La primera dimensión corresponde al interés de amistad y afecto hacia las personas, porque en toda interacción social se intenta buscar la empatía y evitar situaciones y sentimientos que generen hostilidad.

- La segunda dimensión equivale al entusiasmo, una de las señas distintivas de las personas consideradas felices.

- La tercera hace mención a las muestras de cariño. Resulta curioso que la pérdida de entusiasmo se produzca, sobre todo, ante la ausencia o deficitarias muestras de cariño y afecto.

- La cuarta dimensión es el amor proporcionado en el seno familiar, especialmente las relaciones paterno-filiales y fraternales, que constituyen la verdadera fuente de felicidad.

- Y, finalmente, la última dimensión corresponde al trabajo como bien necesario en la sociedad.

A tenor de lo expuesto, podemos argumentar que la felicidad se considera dinámica, con cierta estabilidad temporal y que puede medirse o evaluarse. De este modo, es muy usual que durante muchos momentos de nuestro ciclo vital hagamos balance de nuestras vidas, especialmente ante momentos de profundas crisis o cambios severos. Esta evaluación se vincula a dos dimensiones: la primera al grado de satisfacción percibido ante los acontecimientos acaecidos, y la segunda a la totalidad de las emociones experimentadas, tanto positivas como negativas (Alhatef, 2018).

Como señalan Hervás y Vázquez (2006), tenemos un medidor de bienestar que nos permite analizar y modificar nuestra conducta para alcanzar el grado de satisfacción, alegría, comodidad y estabilidad deseado. Quizás esos cambios de comportamiento se puedan atribuir, como manifiesta Solano (2009) a que el componente cognitivo del bienestar psicológico adquiere una relativa estabilidad temporal que permite al sujeto llevar a cabo una reflexión madurada. Al hilo de lo argumentado, los estudios de Ryan y Deci (2006) establecen que el bienestar y las metas obtenidas constituyen un proceso, un largo camino que debe recorrerse durante la trayectoria del ciclo vital.

Y, para finalizar, no nos extraña, tal y como argumenta Vázquez (2009) basándose en Fredrickson (2006) que la felicidad, entre todos los términos empleados por la psicología positiva, constituya el elemento más significativo para la obtención del deseado índice de bienestar, pues engloba viarias dimensiones: bienestar psicológico o subjetivo, satisfacción vital, áreas de satisfacción, afectos positivos y negativos, placer, estado de ánimo positivo y calidad de vida. 


\section{Bienestar de las personas mayores}

Como tendencia general, se tiene una visión negativa de la senectud. Tal y como afirma Belando (2015), durante esta etapa del ciclo vital el peso de la imagen social negativa de los ancianos todavía es muy considerable.

Es cierto que la vejez suele conllevar determinadas pérdidas propias de la edad y puede afectar a los índices del bienestar personal (Mella y otros, 2004; Molina y Meléndez, 2006; Prieto y otros, 2008). Algunas experiencias en este ciclo vital son la jubilación, la viudez, los cambios de residencia, el detrimento en el grado de participación social, el deterioro cognitivo y motor, la reducción del poder adquisitivo y el acaecimiento de otras circunstancias (Rioseco y otros, 2008).

Afortunadamente, se ha producido un cambio de paradigma en lo que respecta al colectivo de mayores, porque durante los últimos años han proliferado las investigaciones focalizadas exclusivamente en las patologías, declives y déficits. Actualmente, desde la psicogerontología, también cobran suma relevancia las dimensiones vinculadas al bienestar emocional en la vejez (Jiménez e Izal, 2016). No obstante, no debemos establecer generalidades sobre los aspectos negativos, porque Martínez (2016) señala que los ancianos representan un grupo muy heterogéneo, y lo idóneo es tomar en consideración peculiaridades individuales como el género, la edad, el nivel educativo, el estado de salud, etcétera, y que estas personas tienen, además, diferentes intereses y necesidades.

Concerniente al bienestar de los mayores y, por ende, a la experimentación de sentimientos positivos, destacamos la importancia de presentar unos adecuados índices de apoyo social, concepto que según Castellano (2014) puede dividirse en material (recursos monetarios, regalos, alimento, ropa), emocional (acompañamiento, afecto, empatía, preocupación por las necesidades) y cognitivos (información, consejos).

En cambio, Vivaldi y Barra (2012) dividen el apoyo en cuantitativo-estructural, que evalúa la cantidad de vínculos relacionales mantenidos por el individuo; y cualitativo-funcional, que focaliza la atención en las relaciones significativas y en las apreciaciones subjetivas de las mismas (Escobar, Puga y Martín, 2008).

Son evidentes los beneficios de disponer de adecuadas redes de ayuda (Pillemer y otros, 2000), y mantener sólidas redes familiares y sociales resulta extremadamente positivo. Arias (2013) considera que estas redes permiten la provisión de recursos para satisfacer las necesidades, proporcionan sentido de valor social y autoestima, y favorecen la integración y participación comunitaria, por lo que se consideran uno de los factores de mayor incidencia en la calidad de vida de los mayores.

Algunos de los atributos de las redes familiares y sociales son: 
- Permiten combatir los efectos negativos de esta etapa vital, pues a mayor apoyo — formal e informal_, mejores posibilidades de responder a nuevas demandas de la vejez (Arias, 2008).

- Neutralizan el estrés, la soledad y otros sentimientos negativos, y contribuyen a mejorar el bienestar integral y la satisfacción (Aguerre y Bouffard, 2008; Kleinspehn, Kotter y Smith, 2008; Phillips y otros, 2008; Polizzi, 2011).

- Favorecen la reducción de la mortalidad y la mejora en la salud, y propician el incremento y fortalecimiento de las relaciones sociales, vecinales y amicales (Vázquez, Hernangómez y Hervás, 2004).

- Brindan la oportunidad de experimentar afecto, ayuda e información, actuando como un mediador o neutralizador frente a momentos y situaciones estresantes (Yanguas y Arrázola, 2006). En este sentido, los estudios de Ponce y otros (2009) argumentan que la ayuda de carácter emocional, instrumental y afectivo resulta eficaz para proteger la salud.

- Finalmente, para Barra (2004) las redes constituyen un mecanismo eficaz para aumentar la autoestima y la estabilidad, y mejorar los recursos personales.

Quedando constatada la relevancia del apoyo social, y en especial familiar, no nos sorprenden en absoluto los datos obtenidos por Fuentes (2014), que revelan que la familia constituye la principal fuente de apoyo. Aunque destaca que en edades jóvenes esta fuente es la pareja, conforme se incrementa la edad son los propios hijos. Estos datos coinciden con los de Alfonso, Soto y Santos (2016), quienes no dudan en señalar a los sistemas familiares como principales proveedores de bienestar, y en expresar la correlación positiva entre el índice de ayuda percibida y la calidad de vida en los adultos mayores.

Por el contrario, se demuestra que niveles deficitarios de apoyo o su ausencia conllevan trastornos físicos y psicológicos, y merman visiblemente la calidad de vida (Herrero y Gracia, 2005). Al respecto, de acuerdo a las investigaciones de Bozo, Toksabay y Kürüm (2009), las personas que conviven con familiares presentan mejores índices de salud que aquellos que viven solos.

No obstante, los ancianos deben afrontar con optimismo esta nueva fase de la vida, ya que aquellos que adoptan actitudes negativas hacia el envejecimiento mueren antes y enferman más (Levy y otros, 2002).

Vivir positivamente es siempre muy recomendable, más aún cuando en la senectud, a diferencia de otros estadios vitales, el tiempo es limitado e incierto (Carstensen, 2006). Precisamente una de las ventajas que ofrece la tercera edad es disfrutar de la jubilación y realizar actividades de ocio que antaño no pudieron tener. Además, en el caso de ser abuelos pueden pasar más tiempo con sus nietos, sobre todo actualmente, pues a consecuencia de las nuevas dinámicas familiares se les considera titulares activos de las familias (Bravo, Corredor y Campo, 2018). 
Ejercer de abuelo es gratificante y placentero, ya que se experimentan elevados niveles de satisfacción, alegría, felicidad, sentimientos de juventud y utilidad (Osuna, 2006). En este sentido, Marín-Rengifo y Palacio-Valencia (2015) manifiestan que la abuelidad supone una nueva oportunidad de revivir únicamente las experiencias positivas de la crianza, liberando esta vez a los abuelos de las obligaciones y responsabilidades parentales. De otra parte, debemos puntualizar que también se han analizado en profundidad los beneficios de las relaciones intergeneracionales, y estimamos conveniente focalizarnos, aunque sea someramente, en las emociones que conllevan las citadas relaciones, estrechamente vinculadas a los efectos positivos experimentados por abuelos y nietos. En este sentido, Sanz, Mula y Moril (2011) manifiestan que la transmisión de valores adquiere una gran importancia, pues permite consolidar relaciones basadas en la cercanía, el afecto y el carińo, y resultan además esenciales para lograr el correcto desarrollo psicológico y social de los menores. También Pinazo, Bendicho y Company (2008) estiman que el contacto asiduo entre abuelos y nietos fomenta la cooperación y proporciona una comunicación más cercana que permite compartir sentimientos.

\section{Metodología}

Para conocer los determinantes de la felicidad de las personas mayores se utilizó el barómetro del CIS 3109, publicado en septiembre de 2015. Se eligió dicha base de datos pues cuenta con una variable de autopercepción de la felicidad. Se trata de una variable de escala donde el entrevistado valora su felicidad de 0 (completamente infeliz) a 10 (completamente feliz). El barómetro cuenta con las preguntas habituales de los barómetros del CIS: valoración de la situación económica y política del país en el momento de efectuarse el cuestionario y en el último año; principales problemáticas del país y grado de preocupación por las mismas; escala de autoubicación ideológica; religiosidad y frecuencia de asistencia a oficios religiosos. Además, se eligió el barómetro porque incluye preguntas sobre relaciones intergeneracionales que toman en cuenta las relaciones y convivencia con familiares menores de 35 años.

La muestra final está compuesta por un total de 582 individuos. A objeto de conocer los determinantes de la felicidad, en primer lugar se hizo un análisis bivariable a través de comparaciones de medias y correlaciones. Finalmente, para dilucidar el estado conjunto de todas las variables se llevó a cabo un modelo de regresión logística simple en cinco pasos que corresponden a cinco modelos consecutivos. El primer modelo solo tiene en cuenta las características demográficas básicas (estado civil, género y edad); el segundo añade variables socioeconómicas, como el estatus socioeconómico del padre del entrevistado, el estatus socioeconómico del propio 
entrevistado y el nivel máximo de estudios obtenidos; el tercer modelo agrega el estado de salud, y en el cuarto modelo se añaden la autoubicación ideológica y la confesión religiosa. Finalmente, el quinto y último modelo incluye la convivencia y relaciones con menores de 35 años. Se trata de un modelo robusto que pasa de un $\mathrm{R}$ de 0,219 en su primer paso a 0,391 en el quinto paso. Este modelo de regresión logística mide el grado de relación entre las variables, lo que permite observar si crece o disminuye el grado de significación, conforme estas se van introduciendo en los sucesivos pasos. Se confirma un incremento al finalizar el último paso y se determina que las dos variables más significativas son el estado de salud y el estado civil.

\section{Resultados}

En general, se puede afirmar que los mayores de 65 años son personas felices. El valor medio de la escala de felicidad es de 7,1 , esto es, algo menos de medio punto por debajo de la media para toda la población $(7,5)$. La dispersión es también muy similar para ambos conjuntos de población ( 2 y 1,8 puntos respectivamente de desviación típica). Aunque existe una correlación significativa entre la edad y la felicidad, no hay un descenso suficientemente acusado de las personas más jóvenes a las más mayores. La alta desviación típica hace necesario indagar por los factores que determinan un mayor o menor grado de felicidad.

Para conocer la influencia de dichos factores, se llevaron a cabo comparaciones de media mediante ANOVA, así como correlaciones en el caso de las variables numéricas. Los resultados de esos análisis, así como las características de la muestra pueden observarse en la tabla 1. Las variables socioeconómicas que mejor predicen la felicidad son el género, el estado civil, la edad y el estatus socioeconómico. Ser hombre, tener más edad y un estatus socioeconómico alto aumentan el nivel de felicidad. Por otro lado, también es significativo el estado de salud, la distancia hasta la residencia del menor de 35 años más cercano al entrevistado y la autoubicación ideológica. Las dos primeras variables parecen estar relacionadas, pues un mejor estado de salud suele estar asociado a una mayor autonomía y por lo tanto no hace necesaria la presencia cercana de familiares. En cuanto a la ubicación ideológica, aunque sea de forma anecdótica, los datos reflejan un mayor nivel de felicidad entre las personas más conservadoras, que corresponden a sujetos mayores de 65 años, casados y con hijos, estatus socioeconómico medio-alto, con estudios primarios o básicos, que profesan la religión católica y acuden asiduamente a misa y a oficios religiosos, y políticamente son proclives a partidos de derechas. 
Tabla 1. Felicidad personal, medias y correlaciones

\begin{tabular}{|c|c|c|}
\hline \multicolumn{3}{|l|}{ Medias de felicidad personal } \\
\hline & Media & $\mathrm{N}^{\circ}$ \\
\hline Hombre & $7,44^{* *}$ & 246 \\
\hline Mujer & $6,82^{* *}$ & 334 \\
\hline Sin estudios & 7,02 & 124 \\
\hline Primarios & 6,92 & 278 \\
\hline Secundarios & 7,02 & 178 \\
\hline Católico/a & 7,11 & 523 \\
\hline Creyente de otra religión & 6,00 & 4 \\
\hline No creyente & 6,71 & 31 \\
\hline Ateo/a & 7,50 & 14 \\
\hline Casado/a & $7,37^{* *}$ & 365 \\
\hline Soltero/a & $6,94^{* *}$ & 34 \\
\hline Viudo/a & $6,55^{* *}$ & 154 \\
\hline Separado/a & $6,37^{* *}$ & 27 \\
\hline Clase social padre alta & 7,22 & 36 \\
\hline Media & 6,93 & 84 \\
\hline Baja & 7,10 & 455 \\
\hline Estatus socioeconómico alto & $7,44^{*}$ & 168 \\
\hline Medio & $7,20^{*}$ & 111 \\
\hline Bajo & $6,84^{*}$ & 301 \\
\hline Relación <35 Sí & 7,13 & 492 \\
\hline Relación No & 6,77 & 86 \\
\hline Convive $<35$ Sí & 7,07 & 491 \\
\hline Convive No & 7,02 & 88 \\
\hline \multicolumn{3}{|l|}{ Correlaciones } \\
\hline Autoubicación ideológica & $-0,102^{*}$ & 586 \\
\hline Edad & $0,182^{* *}$ & 586 \\
\hline Tiempo tarda familiar $<35$ & $0,093^{*}$ & 497 \\
\hline Estado de salud & $0,176^{* *}$ & 582 \\
\hline
\end{tabular}

Para obtener resultados más concluyentes se llevó a cabo un análisis de regresión logística simple, de modo que se ve el efecto conjunto de la influencia de todas las variables incluidas. Tal y como muestra el análisis (tabla 2), el género deja de predecir significativamente la felicidad una vez que se introduce el estado de salud en el modelo 3. La influencia de la edad deja de ser significativa desde el primer modelo, mien- 
tras que el estado civil se sitúa como uno de los principales factores determinantes, después de incorporarse las escalas político-ideológicas y las relaciones intergeneracionales en los modelos 4 y 5 respectivamente. El estatus socioeconómico, que se introduce en el modelo 2, tan solo es significativo en el segundo paso, y su efecto desaparece con la introducción del estado de salud, siendo precisamente esta variable la de mayor peso en el análisis, pues permanece significativa hasta el último modelo.

Tabla 2. Regresión logística

\begin{tabular}{lccccc}
\hline & Modelo 1 & Modelo 2 & Modelo 3 & Modelo 4 & Modelo 5 \\
\hline Mujer & $-2,587^{*}$ & $-1,408^{*}$ & $-1,408$ & $-1,441$ & $-1,447$ \\
Edad & $-0,233$ & 0,466 & 0,466 & 0,476 & 0,447 \\
Casado/a Sí & $3,577^{* *}$ & $3,424^{* *}$ & $3,424^{* *}$ & $3,402^{* *}$ & $3,325^{* *}$ \\
Ocupación padre & & $-1,611$ & $-1,611$ & $-1,688$ & $-1,641$ \\
ESE & $1,638^{* *}$ & 1,638 & 1,599 & 1,576 \\
Estudios & 0,708 & 0,708 & 0,737 & 0,722 \\
Estado de salud & & & $7,045^{* *}$ & $6,923^{* *}$ & $6,900^{* *}$ \\
Creyente & & & 1,354 & 1,372 \\
Ideología & & & $-1,083$ & $-1,029$ \\
Sí Convive $<35$ & & & & &,- 592 \\
Sí Relación $<35$ & & & & & 0,415 \\
R & & & & & \\
\hline
\end{tabular}

${ }^{*} \mathrm{p}<0,050{ }^{* *} \mathrm{p}<0,010$

Variables que no resultan significativas, además de la edad, señalada anteriormente, son la ocupación del padre, el nivel de estudios, las creencias religiosas y políticas y las relaciones con menores de 35 años.

\section{Conclusiones}

Como ha podido verse en la primera parte de este artículo, el tratamiento de las emociones en las ciencias sociales ha evolucionado de modo que en la actualidad está cada vez más presente en diferentes disciplinas académicas. Las escalas para medir sentimientos o estados de ánimos se han ido extendiendo, y si bien se han aplicado mayoritariamente en el campo de la psicología social y el comportamiento del consumidor, en sociología son escasos los estudios que las aplican. El barómetro del CIS 3109 es una notable excepción que proporciona una escala de autoevaluación de la felicidad que posibilita su análisis. Los resultados obtenidos indican que se trata de una escala que puede ser aplicada para el estudio de tendencias e 
influencias de variables, si bien sería más completo un estudio que incorpore un mayor número de dimensiones.

Estudios teóricos y empíricos previos han incidido en la influencia de un importante número de factores a la hora de determinar el nivel de felicidad, muchos de ellos haciendo hincapié en las redes sociales y de apoyo de las personas mayores. Se trata fundamentalmente de estudios cualitativos que ponían de manifiesto la importancia de las redes sociales en general, y las relaciones intergeneracionales en particular. Al llevar a cabo un análisis para encontrar patrones que confirmen esta evidencia previa, los datos han sido reveladores: las relaciones intergeneracionales en sí mismas no predicen un mayor nivel de felicidad. Sin embargo, sí se confirma una tendencia observada en estudios previos, la importancia del estado civil, que se ha mostrado como predictor no solamente de la felicidad, sino también del estado de salud (Bookwala, Marshall y Manning, 2014; Mirowski, 2017).

Durante este artículo hemos focalizado la atención en los sentimientos positivos que producen las relaciones intergeneracionales. Pero es cierto que en esta etapa de la vida el grado de felicidad puede debilitarse, como señala Flórez (2003), por cuestiones de salud, fallecimiento de amigos y de familiares, y también ante la experimentación de sentimientos negativos tales como angustia, soledad y preocupación ante la proximidad de la muerte. Estas situaciones provocan estigmatización de la senectud, y generan cierto rechazo (Batsch y Mittelman, 2012).

Como hemos indicado, el estado civil constituye una de las variables que predice la felicidad, y cuando acontece la viudez en edades avanzadas genera un detrimento de los contactos sociales, diminución de los ingresos económicos y un evidente empeoramiento del estado de salud (Naef y otros, 2013). Por otra parte, durante la viudez también se acentúan la soledad y el aislamiento social, por lo que el refuerzo de las redes de apoyo primarias constituyen un mecanismo eficaz para evitar el agravamiento de estas situaciones (Black, Holly y Santanello, 2012; Ayuso, 2012).

Una vez más, los resultados ponen de manifiesto la necesidad de implementar políticas que fomenten la autonomía y, por ende, un mejor estado de salud entre las personas mayores, con las consecuencias positivas que de ello se derivan, incluyendo una mayor felicidad.

\section{REFERENCIAS}

Aguerre, C. y Bouffard, L. (2008). Envejecimiento exitoso: Teorías, investigaciones y aplicaciones clínicas. Revista de la Asociación Colombiana de Gerontología y Geriatría, 22, 1146-1162.

Alarcón, R. (2006). Desarrollo de una escala factorial para medir la felicidad. Revista Interamericana de Psicología, 40(1), 95-102. 
Alarcón, R. (2015). La idea de la felicidad. Apuntes. Ciencias Sociales, 5(1). https://doi. org/10.18259/acs.2015002.

Alfonso, L., Soto, D. y Santos, N. A. (2016). Calidad de vida y apoyo social percibido en adultos mayores. Revista de Ciencias Médicas de Pinar del Río, 20, 47-53.

Alhatef, E. (2018). «Bienestar psicológico y apoyo social percibido en adultos mayores». Tesis de Licenciatura. Fundación UADE, Universidad de Buenos Aires.

Arias, C. (2008). El apoyo social en la vejez: Alternativas de acción frente a los desafíos del envejecimiento poblacional. Perspectivas en Psicología, 5(2), 54-61.

Arias, C. (2013). El apoyo social en la vejez: la familia, los amigos y la comunidad. Revista Kairós Gerontología, 16 (4), 313-329.

Arraga, M. V. y Sánchez, M. (2010). Bienestar subjetivo en adultos mayores venezolanos. Interamerican Journal of Psychology, 44(1), 12-18.

Ayuso, L. (2012). Las redes personales de apoyo en la viudedad en Espańa. Revista Española de Investigaciones Sociológicas, 137, 3-24. https://doi.org/10.5477/cis/reis.137.3.

Barra, E. (2004). Apoyo social, estrés y salud. Psicología y Salud, 14(2), 237-243.

Batsch, N. L. y Mittelman, M. S. (2012). World Alzheimer Report 2012: Overcoming The Stigma of Dementia. Londres: Alzheimer's Disease International (ADI).

Belando, M. R. (2015). Conflicto intergeneracional y algunos caminos hacia la sociedad intergeneracional. Prisma Social, 14, 545-573.

Belli, S. e Íñiguez, L. (2008). El estudio psicosocial de las emociones: una revisión y discusión de la investigación actual. PSICO, Porto Alegre, 39(2), 139-151.

Bericat, E. (2012). Emociones. Sociopedia.isa. https://doi.org/ 10.1177/205684601261.

Beytía, P. y Calvo, E. (2011). ¿Cómo medir la felicidad? Claves de Políticas Públicas, 4. https://doi.org/10.2139/ssrn.2302809.

Black, H. K., Holly, R. y Santanello, B. A. (2012). The Salience of Family Wordview in Mourning an Elderly Husband and Father. The Gerontologist, 52(4), 472-483. https://doi.org/10.1093/geront/gnr148.

Bookwala, J., Marshall, K. I. y Manning, S. W. (2014). Who needs a friend? Marital status transitions and physical health outcomes in later life. Health Psychology, 33(6), 505. https://doi.org/10.1037/hea0000049.

Bozo, O., Toksabay, N. y Kürüm, O. (2009). Activities of daily living, depression, and social support among elderly Turkish people. Journal of Psychology, 143, 193-205. https://doi.org/10.3200/JRLP.143.2.193-206.

Bravo, J. E., Corredor, Z. B. y Campo, H. E. (2018). Las características de las relaciones intergeneracionales abuelo-nieto en 80 familias del colegio Rafael Uribe de la Localidad de Ciudad Bolivar de Bogotá D. C. Bogotá: Universidad de la Salle.

Brown, S. D. (2005). Collective Emotions: Artaud's Nerves. Culture and Organization, 11(4), 235-247. https://doi.org/10.1080/14759550500361363.

Burke, P. J. y Stets, J. E. (2009). Identity Theory. Nueva York: Oxford University Press. https:/doi.org/10.1093/acprof:oso/9780195388275.001.0001

Carstensen, L. L. (2006). The influence of a sense of time on human development. Science, 312, 1913-1915. https://doi.org/10.1126/science.1127488. 
Castellano, C. L. (2014). La influencia del apoyo social en el estado emocional y las actitudes hacia la vejez y el envejecimiento en una muestra de ancianos. International Journal of Psychology and Psychological Therapy, 14(3), 365-377.

Damasio, A. (2006). El error de Descarte. La emoción, la razón y el cerebro humano. Barcelona: Crítica.

Damasio, A., Grabowski, J. T., Bechara, A., Damasio, H., Ponto, L. L., Parvizi, J. y Hichwa, R. D. (2001). Subcortical and cortical brain activity during the feeling of selfgenerated emotions- Nature Neuroscience, 3, 1049-1056. https://doi.org/ 10.1038/79871.

Denzin, N. K. (2009) [1984]. On Understanding Emotion. New Brunswick, NJ: Transaction.

Diener, E. (2000). Subjective well-being: The science of happiness, and a proposal for a national index. American Psychologist, 55, 34-43. https://doi. org/10.1037/0003-066X.55.1.34

Edwards, D. (1999). Emotion discourse. Culture and Psychology, 5(3), 271-291. https://doi. org/10.1177/1354067X9953001

Escobar, M. A., Puga, D. y Martín, M. (2008). Asociaciones entre la red social y la discapacidad al comienzo de la vejez en las ciudades de Madrid y Barcelona en 2005. Revista Española de Salud Pública, 82, 637-651. https://doi.org/10.1590/ S1135-57272008000600005

Fernández, A. M. (2011). Antropología de las emociones y teoría de los sentimientos. Revista Versión. Nueva Época, 26.

Flórez, J. A. (2003). Felicidad y salud. Salud Global, 3(1).

Filliozat, I. (2007). El corazón tiene sus razones. Conocer el lenguaje de las emociones. Barcelona: Urano.

Fredrickson, B. L. (2006). The Broaden and Build Theory of Positive Emotions. En M. Csikszentmihalyi y S. Csikszentmihalyi (eds.), A life worth living. Contributions to Positive Psychology (pp. 85-103). Oxford: Oxford University Press.

Fuentes, C. L. C. (2014). La influencia del apoyo social en el estado emocional y las actitudes hacia la vejez y el envejecimiento en una muestra de ancianos / The influence of social support on the emotional state and attitudes towards old age and ageing in a sample of elderly. Revista Internacional de Psicología y Terapia Psicológica, 14, 365-377.

Good, M. D., Good, B. J. y Fischer, M. M. J. (1988). Introduction: Discourse and the study of emotion, illness and healing. Culture, Medicine and Psychiatry, 12(1), 1-7. https://doi.org/10.1007/BF00047035.

Gross, J. J. y Feldman, L. (2011). Emotion Generation and Emotion Regulation: One or Two Depends on Your Point of View. Emotion Review, 3(1), 8-16. https://doi. org/10.1177/1754073910380974.

Guedes, S. M. y Álvaro, J. L. (2010). Naturaleza y cultura en el estudio de las emociones. Revista Española de Sociología, 13, 31-47.

Haidt, J. (2007). La hipótesis de la felicidad. La búsqueda de verdades modernas en la sabiduría antigua. Barcelona: Gedisa.

Harré, R. y Stearns, P. N. (1995). Discursive psychology in practice. Londres: Sage. 
Herrero, J. y Gracia, E. (2005). Redes sociales de apoyo y ajuste biopsicosocial en la vejez: un análisis comparativo en los contextos comunitario y residencial. Intervención Psicosocial, 14, 41-50.

Hervás, G. y Vázquez, C. (2006). La regulación afectiva: modelos, investigación e implicaciones para la salud mental y física. Revista de Psicología General y Aplicada, 59, 9-32.

Hochschild, A. R. (1990). Ideology and Emotion Management: A Perspective and path for future research. En T. D. Kemper (Ed.), Research Agenda in the Sociology of Emotions (pp. 117-142). Albany, NY: University of New York Press.

Íniguez L. (2005). Nuevos debates, nuevas ideas y nuevas prácticas en la psicología social de la era 'post construccionista'. Athenea Digital, 8. https://doi.org/10.5565/rev/ athenead/v1n8.235

Jasper, J. M. (2011). Emotions and social movements: Twenty years of theory and research. Annual Review of Sociology, 37, 285-303. https://doi.org/ 10.1146/ annurev-soc-081309-150015.

Jiménez, M. G. y Izal, M. (2016). Programa para la mejora del bienestar de las personas mayores. Estudio piloto basado en la psicología positiva. Suma Psicológica, 23(1), 51-59. https://doi.org/10.1016/j.sumpsi.2016.03.001.

Kleinspehn, A., Kotter, D. y Smith, J. (2008). Self-perceptions of aging: Do subjective age and satisfaction with aging change during old age?. Journal of Gerontology, 63, 377385. https://doi.org/10.1093/geronb/63.6.P377.

Lawler, E. J. (2001). An affect theory of social exchange. The American Journal of Sociology, 107(2), 321-352. https://doi.org/10.1086/324071.

Lawler, E. J., Thye, S. R. y Yoon, J. (2008). Social Exchange and micro social order. American Sociological Review, 73, 519-542. https://doi.org/10.1177/000312240807300401.

Levy, B. R., Slade, M. D., Kunkel, S. R. y Kasl, S. V. (2002). Longevity increased by positive self-perceptions of agings. Journal of Personality and Social Psychology, 83, 261-270.

Luna, R. (2010). La sociología de las emociones como campo disciplinario. Interacciones y estructuras sociales. En A. Scribano y P. Lisdero (coords.), Sensibilidades en Juego: miradas múltiples desde los estudios sociales de los cuerpos y las emociones. Córdoba: CEA-CONICET.

Marín, A. L. y Palacio, M. C. (2015). El abuelazgo: enlace intergeneracional en la crianza y cuidado de la primera infancia. Revista Latinoamericana de Estudios de Familia, 7, 11-27. https://doi.org/10.17151/rlef.2015.7.2.

Marina, J. A. (2006). El laberinto sentimental. Barcelona: Anagrama.

Martínez, P. (2016). «Mayores y bienestar personal». Trabajo de fin de grado. Universidad de Cádiz.

McMahon, D. M. (2006). Una historia de la felicidad. Madrid: Taurus.

Mella, R., González, L., D’ Appolonio, J., Maldonado, I., Fuenzalida, A. y Díaz, A. (2004). Factores asociados al bienestar subjetivo en el adulto mayor. Psykhe, 13, 79-89. https://doi.org/10.4067/S0718-22282004000100007.

Michael, M. (2006). Technoscience and everyday life: The complex simplicities of the mundane. Nueva York: Open University Press. 
Mirowsky, J. (2017). Education, social status and health. Routledge. https://doi. org/10.4324/9781351328081

Molina, C. y Meléndez, J. (2006). Bienestar psicológico en envejecientes de la República Dominicana. Geriátrika, 22, 97-105.

Naef, R., Ward, R., Mahrer-Imhof, R. y Grande, G. (2013). Characteristics of the bereavement experience of older persons after spousal loss: An integrative review. International Journal Nursery Studies, 50(8), 1108-1121. https://doi.org/10.1016/j. ijnurstu.2012.11.026.

Osuna, M. J. (2006). Relaciones familiares en la vejez: vínculos de los abuelos y las abuelas con sus nietos y nietas en la infancia. Revista Multidisciplinar de Gerontología, 16(1), 16-25.

Phillips, D. R., Sui, O. L., Yeh, A. G. y Cheng, K. H. (2008). Informal social support and older person's psychological well being in Hong Kong. Journal of Cross-Cultural Gerontology, 23(1), 39-55. https://doi.org/10.1007/s10823-007-9056-0

Pillemer, K., Moen, P., Wethington, E. y Glasgow, N. (2000). Social Integration in the Second Half of Life. Baltimore, MD: Johns Hopkins University Press.

Pinazo, S., Bendicho, J. y Company, R. (2008). Cómo conectar el cine con las relaciones intergeneracionales. Valencia: Universidad de Valencia.

Polizzi, L. (2011). La importancia de la pareja, los hijos y los amigos en la vejez. En Primeras Jornadas de Psicología. Los desafíos de la clínica actual. Mar de Ajó, Buenos Aires: Universidad Atlántida Argentina.

Ponce, J. M., Velázquez, A., Márquez, E., López, L. y Bellido, M. L. (2009). Influencia del apoyo social en el control de las personas con diabetes. Index de Enfermería, 18(4), 224228. https://doi.org/10.4321/S1132-12962009000400002

Prieto, M. E., Fernández, G., Rojo, F., Lardiés, R., Rodríguez, V. y Ahmed, K. (2008). Factores sociodemográficos y de salud en el bienestar emocional como dominio de calidad de vida de las personas mayores en la Comunidad de Madrid: 2005. Revista Española de Salud Pública, 82, 301-313. https://doi.org/10.1590/S1135-57272008000300006

Rioseco, R., Quezada, M., Ducci, M. y Torres, M. (2008). Cambio en las redes sociales de adultos mayores beneficiarios de programas de vivienda social en Chile. Revista Panamericana de Salud Pública, 23, 147-153. https://doi.org/10.1590/ S1020-49892008000300001

Russell, B. (2004). La conquista de la felicidad. Barcelona: Debolsillo.

Ryan, R. M. y Deci, E. L. (2006). Self-regulation and the problem of human autonomy: does psychology need choice, self-determination, and will?. Journal of personality, 74, 1557-1586. https://doi.org/10.1111/j.1467-6494.2006.00420.x

Sanz, R., Mula, J. M. y Moril, R. (2011). La relación abuelos-nietos-escuela: una excusa o una necesidad. En XII Congreso Internacional de Teoría de la Educación. Barcelona. https://www.cite2011.com/Comunicaciones/Familias/142.pdf

Scribano, A. (2012). Sociología de los cuerpos/emociones. Revista Latinoamericana de Estudios sobre Cuerpos, Emociones y Sociedad, 4(10), 91-111. 
Smith, H. y Schneider, A. (2009). Critiquing Models of Emotions. Sociological Methods and Research, 37(4), 560-589. https://doi.org/10.1177/0049124109335790

Solano, A. C. (2009). El bienestar psicológico: cuatro décadas de progreso. Revista Interuniversitaria de Formación del Profesorado, 66, 43-72.

Stryker, S. (2004). Integrating emotion into identity theory. Advances in Group Processes, 21, 1-23. https://doi.org/10.1016/S0882-6145(04)21001-3

Vázquez, C. (2009). La ciencia del bienestar psicológico. En C. Vázquez y G. Hervás (coords.), La ciencia del bienestar (pp. 6-27). Madrid: Alianza.

Turner, J. H. (1999). Toward a general sociological theory of emotions. Journal for the Theory of Social Behaviour, 29(2), 133-162. https://doi.org/10.1111/1468-5914.00095

Vázquez, C., Hernangómez, L. y Hervás, G. (2004). Longevidad y emociones positivas. En L. Salvador, A. Cano y J. R. Cabo (eds.), Longevidad. Tratado integral sobre la salud en la segunda mitad de la vida. Madrid: Panamericana.

Vivaldi, F. y Barra, E. (2012). Bienestar psicológico, apoyo social percibido y percepción de salud en adultos mayores. Terapia Psicológica, 30(2), 23-29. https://doi.org/10.4067/ S0718-48082012000200002.

Yanguas, J. y Arrázola, F. J. L. (2006). Intervención psicosocial con personas mayores. Madrid: Alianza. 